\section{Estrutura e processo de trabalho na prevenção do câncer de colo de útero na Atenção Básica à Saúde no Brasil: Programa de Melhoria do Acesso e da Qualidade - PMAQ}

\section{Structure and work process in the prevention of cervical cancer in Health Basic Attention in Brazil: Program for the Improvement of Access and Quality}

Elaine Tomasi 1

Talita Fischer Oliveira 2

Pedro Agner Aguiar Fernandes 3

Elaine Thumé 4

Denise Silva da Silveira 5

Fernando Vinholes Siqueira 6

Suele Manjourany Silva Duro 7

Mirelle de Oliveira Saes 8

Bruno Pereira Nunes 9

Anaclaudia Gastal Fassa 10

Luiz Augusto Facchini 11

1, 10, 11 Programa de Pós-Graduação em Epidemiologia. Departamento de Medicina Social. Universidade Federal de Pelotas. Av. Duque de Caxias, 250. Fragata. Pelotas, RS, Brasil. CEP: 96.030-000. E-mail: tomasiet@gmail.com

2-9 Universidade Federal de Pelotas. Pelotas, RS, Brasil.

\section{Resumo}

Objetivos: descrever e analisar a adequação da estrutura e processo das equipes do Programa de Melhoria do Acesso e da Qualidade (PMAQ-AB) na prevenção do câncer de colo de útero na Atenção Básica no Brasil.

Métodos: estudo transversal conduzido em todas as unidades básicas de saúde (UBS), entre 2012 e 2013. Estimou-se a prevalência da adequação da estrutura (insumos/materiais) e do processo de trabalho para o exame de Papanicolau. A associação da adequação com características das unidades $e$ equipes foi estimada pela razão de prevalência $(R P) e$ intervalos de confiança de 95\% (IC95\%).

Resultados: foram estudadas 38.812 UBS e 17.202 equipes de saúde. A prevalência de adequação da estrutura e do processo de trabalho foi $49 \%$ e $30 \%$, respectivamente. A adequação da estrutura e o processo estiveram associados ao maior porte do municipio e IDH. A prevalência de adequação da estrutura foi maior nas unidades da Estratégia de Saúde da Família (RP=1,35; IC95\%:1,33-1,38), cuja adequação do processo de trabalho esteve associada à população adscrita $<4.000$ pessoas e equipe mínima completa.

Conclusões: a estrutura e o processo de trabalho para o rastreamento do câncer de colo uterino necessitam ser aprimoradas. As equipes de saúde carecem de maior qualificação das ações relacionadas a esta ação programática.

Palavras-chave Atenção primária à saúde, Estrutura dos serviços, Estratégia saúde da família, Neoplasias do colo do útero, Saúde da mulher 


\section{Introdução}

O câncer de colo do útero é uma das neoplasias com maior incidência e maiores taxas de mortalidade entre as mulheres. Em 2012, foram detectados 527.624 novos casos e registradas 265.653 mortes em todo o mundo. ${ }^{1}$ No Brasil, foram registrados 17.540 casos novos de câncer de colo, observandose uma incidência de 17 casos por 100.000 no ano de 2012. Essa incidência é maior do que a observada nos Estados Unidos e Canadá, que é de cerca de 6 por 100.000 e menor do que a da Argentina, que é de 21 por 100.000. A taxa de letalidade de $30 \%, 2$ é igualmente elevada, constituindo a terceira causa de morte por câncer em mulheres brasileiras. A taxa de mortalidade em 2012 foi de 4,7 óbitos por 100.000 mulheres, já ajustada para a população mundial.2 É uma doença curável desde que detectada e tratada precocemente. De acordo com o Instituto Nacional do Câncer, a taxa de cura varia de $85 \%$ a $100 \%{ }^{3}$

No entanto, a redução nas taxas de incidência e mortalidade depende tanto de uma alta cobertura do exame preventivo, de no mínimo $80 \%$ da população feminina em idade de risco, quanto da qualidade dos procedimentos de coleta, da agilidade dos resultados e do tratamento oportuno. 4

O exame citopatológico de Papanicolau é o teste utilizado para detectar lesões precursoras do câncer de colo de útero, devido à elevada acurácia e efetividade quando aplicado em programas de rastreamento. $5 \mathrm{O}$ teste é realizado com rapidez e possui um custo unitário relativamente baixo, valor menor do que oito reais ou três dólares, de acordo com a Tabela de Procedimentos Ambulatoriais do Sistema Único de Saúde. 6 O exame é considerado um meio diagnóstico efetivo de prevenção, devido à sua elevada especificidade, reduzindo as chances de tratamentos e intervenções desnecessárias. ${ }^{3,7}$ Devido a tais características, o exame de Papanicolau é um teste útil para uso em larga escala. ${ }^{8}$

Em 1986, com o projeto "Expansão da Prevenção e Controle do Câncer Cervicouterino" do Programa de Oncologia (PRO-ONCO), ${ }^{7}$ elaborado pelo Instituto Nacional do Câncer, houve um reconhecimento por parte das autoridades brasileiras das elevadas incidência e mortalidade por câncer de colo uterino. Dessa forma, políticas públicas vêm sendo instituídas para diminuir a incidência e a mortalidade da doença, como programas de rastreamento, diagnóstico e tratamento, com ênfase em ações na rede de atenção básica à saúde. Neste âmbito, é alto o potencial de disseminação da informação à população sobre o rastreamento, de identificação de mulheres na faixa etária prioritária e com risco aumentado, de convocação para realização da coleta da citologia e de identificação de faltosas e consequente busca ativa. ${ }^{8}$

No âmbito das unidades básicas de saúde (UBS), a população tem maior acesso à informação sobre o rastreamento. Além disso, as equipes de saúde têm maior facilidade para a identificação de mulheres na faixa etária elegível para o programa e as com maior risco de desenvolvimento do evento, além da convocação das mulheres para a realização dos exames periódicos e da busca das faltosas. ${ }^{8}$

No Brasil, levantamentos têm mostrado o aumento do percentual de mulheres submetidas a pelo menos um exame de Papanicolau, que passou de $82,6 \%$, em 2003 para 87,1\% em 2008. ${ }^{9}$ Sabe-se que o percentual de cobertura do teste é influenciado por características das usuárias, como o nível socioeconômico e idade, 9,10 além das características dos serviços, com destaque para a qualificação das equipes da Atenção Básica (AB) e os oportunos e adequados procedimentos técnicos relacionados ao exame nos demais serviços do sistema de saúde.7

A partir de 2012, o Ministério da Saúde (MS) implantou o Programa de Melhoria do Acesso e da Qualidade da Atenção Básica (PMAQ-AB), com o objetivo de induzir a ampliação do acesso da população aos serviços, a melhoria das condições de trabalho e da qualidade da atenção, além de investimentos no desenvolvimento dos trabalhadores da AB. ${ }^{11}$ As equipes são incluídas no Programa mediante adesão da gestão municipal que, após processo de avaliação externa, passam a ser certificadas progressivamente e contam com repasse de recursos em função de seu desempenho. Um dos indicadores de qualidade, no âmbito da saúde da mulher, é a cobertura do exame citopatológico (CP) para detecção precoce do câncer de colo de útero. ${ }^{11}$

Este artigo descreve a estrutura e o processo de trabalho para a realização do exame citopatológico de Papanicolau na rede pública de AB. Também buscou-se analisar as características das unidades de saúde e das equipes, e evidências de fatores associados à adequação da estrutura e do processo de trabalho, com vistas à prevenção do câncer de colo de útero, no âmbito do Programa de Melhoria do Acesso e da Qualidade da Atenção Básica (PMAQ).

\section{Métodos}

Trata-se de um estudo de delineamento transversal que integra a avaliação externa do PMAQ realizada por quarenta e uma instituições brasileiras de ensino superior (IES) e lideradas por seis delas: Fundação Osvaldo Cruz (FIOCRUZ), Universidade Federal da 
Bahia (UFBA), Universidade Federal de Minas Gerais (UFMG), Universidade Federal de Pelotas (UFPEL), Universidade Federal do Rio Grande do Sul (UFRGS) e Universidade Federal do Rio Grande do Norte (UFRN). A coleta de dados ocorreu entre os meses de junho de 2012 e março de 2013 e envolveu cerca de 1000 entrevistadores e supervisores em todas as Unidades da Federação.

Durante a pesquisa, foi realizado o censo de todas as UBS registradas no Cadastro Nacional de Estabelecimentos de Saúde, localizadas na zona urbana e rural nos 5565 municípios do Brasil, além do Distrito Federal.12 O instrumento de coleta de dados da avaliação externa do PMAQ-AB foi composto por três módulos: Módulo I - Observação na Unidade de Saúde (UBS); Módulo II - Entrevista com um profissional sobre o processo de trabalho da Equipe de Atenção Básica e verificação de documentos na UBS; Módulo III - Entrevista com usuário na unidade de Saúde (dados não utilizados neste artigo).

As informações sobre a estrutura do serviço para o desenvolvimento das ações de prevenção foram obtidas por meio da aplicação do Módulo I do instrumento de coleta e foram relativas à disponibilidade no serviço dos seguintes insumos/materiais: mesa de exame ginecológico, foco de luz, espéculo descartável, escovinha endocervical, espátula de Ayre, fixador de lâmina, lâmina de vidro e ficha de requisição do Sistema de Informações do Câncer do Colo do Útero (Siscolo). A estrutura do serviço foi classificada adequada se possuísse os oito insumos e materiais acima listados, de acordo com o definido pelas áreas técnicas do MS. 8

Do Módulo II do instrumento foram utilizadas as seguintes questões: a unidade de saúde possui protocolo para priorização de usuárias para o programa de controle do câncer de colo de útero, a equipe coleta material para exame citopatológico, possui registro das coletas e dos exames alterados, realiza o seguimento das mulheres pós-tratamento e realiza ações de divulgação e de sensibilização da população-alvo para o exame. $\mathrm{O}$ processo de trabalho foi considerado adequado quando havia resposta positiva a todos os itens questionados.

Analisou-se a associação entre a adequação da estrutura do serviço de saúde (Módulo I) com as seguintes características: modelo da UBS (Unidade de Saúde da Família - USF/Unidade tradicional), a existência de equipes participantes do PMAQ na UBS ( $\operatorname{sim} /$ não), a região geopolítica (Norte, Nordeste, Centro-Oeste, Sudeste e Sul); o porte do município12 (até 10.000 habitantes; de 10.001 a
30.000 ; de 30.001 a 100.000 ; de 100.001 a 300.000 e mais de 300.000 habitantes), o Índice de Desenvolvimento Humano Municipal (IDH-M)13 classificado em quartis (de 0,467 a 0,642 ; de 0,643 a 0,730 ; de 0,731 a 0,787 e de 0,788 até 0,919 ) e a cobertura populacional da Estratégia de Saúde da Família (ESF) em cada município11 (até $50 \%$; de 50,1 a $75 \%$; de 75,1 a $99,9 \%$ e de $100 \%$ ).

Para a análise sobre o processo de trabalho (Módulo II), além das variáveis citadas anteriormente, utilizou-se a população sob responsabilidade da equipe (até $4.000 \mathrm{hab} / \mathrm{mais}$ de 4.000 hab. $)^{14}$ e possuir equipe mínima da ESF completa, de acordo com a Portaria ${ }^{\circ} 2.488$, de 21 de outubro de 2011- um médico, um enfermeiro, um auxiliar ou técnico de enfermagem e quatro agentes comunitários de saúde ( $\operatorname{sim} /$ não). Uma vez que todas as equipes haviam aderido ao Programa PMAQ, esta variável não foi utilizada na análise.

Os dados foram coletados em formulários eletrônicos disponibilizados em tablets, com envio automatizado ao servidor central do MS. A análise de consistência do banco de dados ficou a cargo de cada uma das seis instituições que lideraram a coleta de dados, sob a coordenação do Departamento de Atenção Básica do MS.

O pacote estatístico Stata 12.0 foi utilizado na análise dos dados. Descreveu-se a distribuição de frequência das unidades de saúde segundo as características investigadas. A prevalência da adequação da estrutura e do processo de trabalho foi estimada de acordo com as características sociodemográficas e do serviço. A análise dos fatores associados à adequação da estrutura e processo de trabalho foi realizada pelo cálculo da Razão de Prevalência (RP), e seus respectivos intervalos de confiança de 95\% (IC95\%). As categorias de referência das variáveis independentes nas análises bivariadas foram as que tiveram menor prevalência, quando as variáveis eram dicotômicas, e as com menor valor, quando as variáveis eram ordinais.

O estudo foi aprovado pelo Comitê de Ética em Pesquisa da Faculdade de Medicina da Universidade Federal de Pelotas sob o ofício 38/12 de 10 de maio de 2012. Todos os participantes assinaram Termo de Consentimento Livre e Esclarecido. Os autores declaram não haver conflitos de interesse em relação ao tema de estudo.

\section{Resultados}

Um total de 38.812 unidades de saúde identificadas no censo respondeu ao instrumento do Módulo I 
(relativo à estrutura). Dentre estas, 29.971 (77\%) eram unidades da ESF e 13.843 (36\%) possuíam equipes participantes do PMAQ.

A Tabela 1 apresenta a distribuição de frequência das unidades e equipes avaliadas segundo características relativas à assistência de saúde. As regiões Nordeste e Sudeste concentraram a maior parte das UBS avaliadas (38\% e 31\%, respectivamente). Quase metade das UBS loca-lizavam-se em municípios com menos de 30.000 habitantes e 14\% situ- avam-se em municípios acima de 300.000 habitantes. Cerca de um quarto das unidades de saúde localizavam-se em municípios com baixo IDH entre 0,467 a 0,642 - e no último quartil de IDH entre 0,788 a 0,919 . No que diz respeito à cobertura da ESF, destaca-se que $28 \%$ estavam em municípios com $100 \%$ de sua população coberta e outros $31 \%$ das UBS localizavam-se em municípios com muito baixas coberturas - inferiores a $50 \%$ (Tabela 1 ).

Tabela 1

Distribuição das unidades básicas de saúde e das equipes avaliadas de acordo com as variáveis independentes. Avaliação externa do PMAQ-AB, Brasil, 2012.

\begin{tabular}{|c|c|c|c|c|}
\hline \multirow[t]{2}{*}{ Variável } & \multicolumn{2}{|c|}{ Unidades de Saúde } & \multicolumn{2}{|c|}{ Equipes } \\
\hline & $\mathrm{n}$ & $\%$ & $\mathrm{n}$ & $\%$ \\
\hline \multicolumn{5}{|l|}{ Modelo de atenção* } \\
\hline Saúde da Família & 29.971 & 77,2 & 16.643 & 96,8 \\
\hline Tradicional & 8841 & 22,8 & 542 & 3,2 \\
\hline \multicolumn{5}{|c|}{ US participante do PMAQ\# } \\
\hline $\operatorname{Sim}$ & 13.843 & 35,7 & - & - \\
\hline Não & 24.969 & 64,3 & - & - \\
\hline \multicolumn{5}{|c|}{ População adscrita à equipe (hab.) } \\
\hline Até 4000 & - & - & 12.730 & 78,2 \\
\hline Mais de 4000 & & & 3552 & 21,8 \\
\hline \multicolumn{5}{|c|}{ Equipe mínima de SF completa\#\# } \\
\hline Sim & - & - & 15.007 & 87,2 \\
\hline Não & - & - & 2195 & 12,8 \\
\hline \multicolumn{5}{|l|}{ Região } \\
\hline Norte & 3210 & 8,3 & 1016 & 5,9 \\
\hline Nordeste & 14.638 & 37,7 & 5525 & 32,3 \\
\hline Sudeste & 11.943 & 30,7 & 6554 & 38,3 \\
\hline Sul & 6315 & 16,3 & 2913 & 17,0 \\
\hline Centro-Oeste & 2706 & 7,0 & 1104 & 6,5 \\
\hline \multicolumn{5}{|c|}{ Porte populacional (hab.) } \\
\hline Até 10.000 & 6119 & 15,8 & 2563 & 15,0 \\
\hline 10.001 a 30.000 & 12.362 & 31,9 & 4741 & 27,7 \\
\hline 30.001 a 100.000 & 10.104 & 26,0 & 3557 & 20,8 \\
\hline 100.001 a 300.000 & 4907 & 12,6 & 2054 & 12,0 \\
\hline Mais de 300.000 & 5320 & 13,7 & 4197 & 24,5 \\
\hline \multicolumn{5}{|l|}{ IDH-M\& (quartis) } \\
\hline 0,467 a 0,642 & 9832 & 25,4 & 3164 & 18,9 \\
\hline 0,643 a 0,730 & 9641 & 24,8 & 3812 & 22,7 \\
\hline 0,731 a 0,787 & 9654 & 24,9 & 3732 & 22,2 \\
\hline 0,788 a 0,919 & 9680 & 24,9 & 6072 & 36,2 \\
\hline \multicolumn{5}{|l|}{ Cobertura de ESF $(\%)$} \\
\hline Até 50 & 12.024 & 31,0 & 4875 & 28,5 \\
\hline 50,1 a 75 & 8322 & 21,4 & 4323 & 25,3 \\
\hline 75,1 a 99,9 & 7703 & 19,8 & 3309 & 19,4 \\
\hline 100 & 10.763 & 27,8 & 4583 & 26,8 \\
\hline Total & 38.812 & 100,0 & 17.202 & 100,0 \\
\hline
\end{tabular}

\# Informação disponível apenas para as US - Módulo I do instrumento;

\#\# 1 Médico + 1 enfermeiro +1 auxiliar ou técnico de enfermagem + 4 agentes comunitários de saúde - Informação disponível apenas para as equipes - Módulo II do instrumento;

* Unidade de Saúde com Estratégia de Saúde da Família;

\& Índice de Desenvolvimento Humano Municipal. 
Um total de 17.202 equipes de saúde, atuando em 13.843 UBS, estavam inscritas no PMAQ e responderam ao instrumento do módulo II, das quais 97\% eram da ESF.

Mais de $20 \%$ das equipes tinham mais de 4000 habitantes sob sua responsabilidade e $88 \%$ contavam com a composição mínima de profissionais preconizada pelo MS (Tabela 1).

Do total de equipes avaliadas, $38 \%$ era da Região Sudeste e 32\% da Região Nordeste. Mais da metade pertencia a municípios com população superior a 30.000 habitantes e com IDH acima de 0,730. Praticamente um quarto das equipes era de municípios com $100 \%$ de cobertura da ESF (Tabela 1).

Quanto à disponibilidade de equipamentos e insumos, observou-se que $83 \%$ das UBS apresentavam mesa de exame ginecológico e foco de luz, $82 \%$ de escova cervical e espátula de Ayre, $81 \%$ dispunha de lâminas, $78 \%$ ficha de requisição para o Siscolo, em $77 \%$ havia espéculo e em $69 \%$ havia fixador (Figura 1).

Para o conjunto das unidades de saúde, a proporção de adequação da estrutura de equipamentos e insumos para a realização do exame citopatológico foi de $49 \%$ ( $n=19.126)$, ou seja, metade dos serviços de saúde dispunha de todos os oito itens.

Considerando os itens avaliados sobre processo de trabalho (Módulo II do instrumento), 97\% coletava material cérvico-uterino para o exame, $92 \%$ realizava ações de divulgação e de sensibilização da população-alvo para o exame, $89 \%$ dispunha de protocolo para priorização de usuárias, $88 \%$ realizava seguimento de mulheres pós-tratamento, $83 \%$ mantinha registro das coletas e dos exames alterados e $45 \%$ registrava as coletas e exames (Figura 2 ).

\section{Figura 1}

Unidades de saúde (\%) segundo a disponibilidade de equipamentos e insumos para realização de exame preventivo ao câncer de colo de útero. ( $n=38.812)$. Avaliação externa do PMAQ-AB, Brasil, 2012.

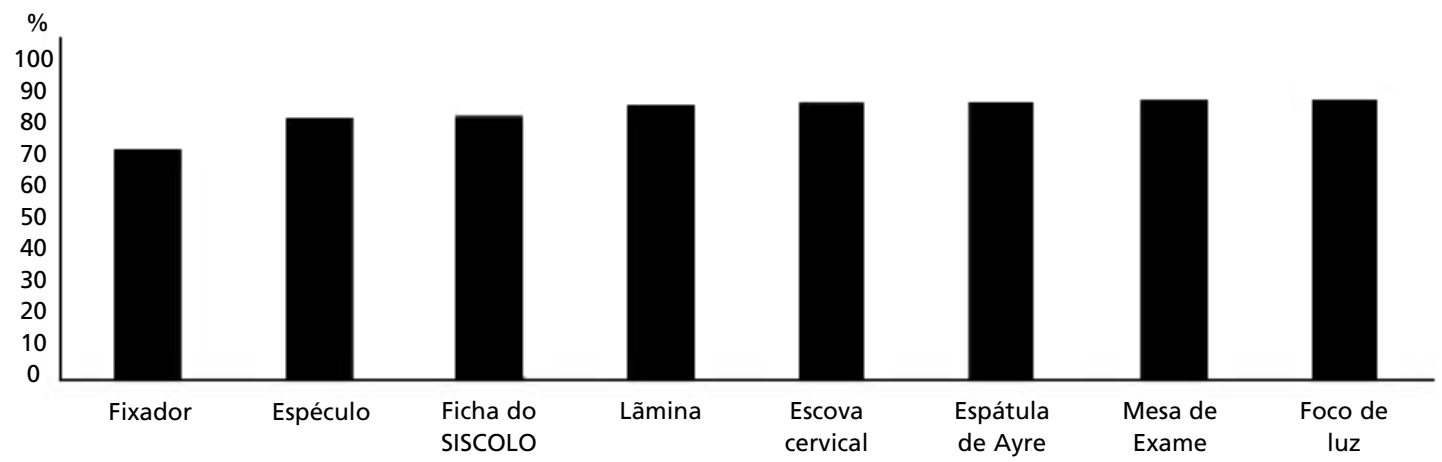

Insumo / Equipamento

\section{Figura 2}

Equipes de saúde (\%) segundo o processo de trabalho para realização de exame preventivo ao câncer de colo de útero $(n=17.202)$. Avaliação externa do PMAQ-AB, Brasil, 2012.

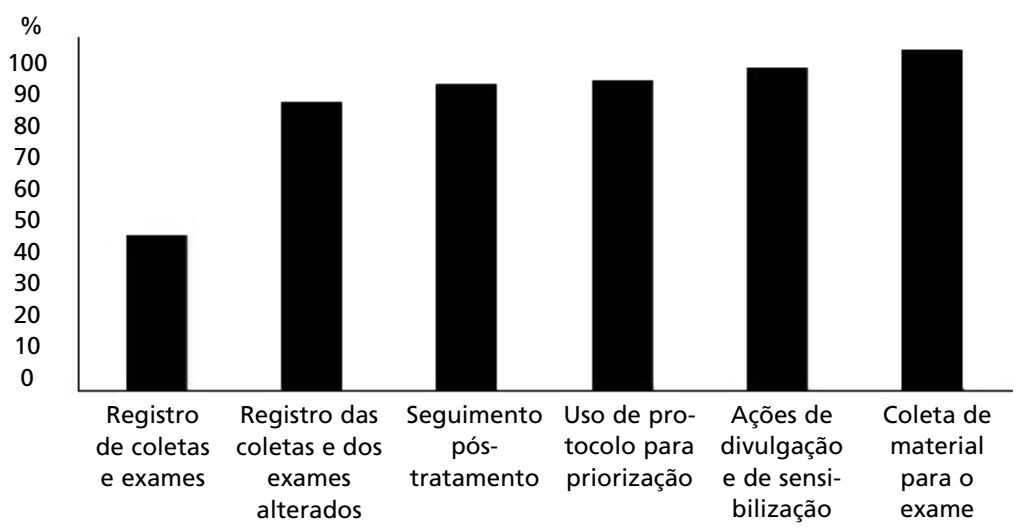

Atividade realizada pela equipe 
Um percentual de $30 \%$ das equipes confirmou a realização de todas as atividades previstas pelo PMAQ quanto ao processo de trabalho e foram classificadas como adequadas (Tabela 2).

As unidades com ESF apresentaram 35\% mais adequação de estrutura do que as unidades tradicionais. Do total, observou-se que o percentual de unidades com estrutura classificada como adequada foi maior naquelas que haviam aderido ao PMAQ $(61 \%)$ do que as que não participavam (43\%) (Tabela 2).

A análise das características associadas à adequação da estrutura mostrou que o percentual de unidades com estrutura adequada variou entre as regiões, sendo cerca de $40 \%$ na Região Norte, e de cerca de $60 \%$ nas Regiões Nordeste, Sul e CentroOeste. Os municípios de maior porte populacional apresentaram significativamente maior adequação da estrutura do que os de menor porte. Quanto maior o IDH dos municípios, mais adequada era a estrutura para realização do exame. E quanto mais altas as coberturas de ESF, menos adequadas estavam as unidades de saúde (Tabela 2).

Quanto à análise das características relacionadas à adequação do processo de trabalho, não foram observadas diferenças na prevalência de adequação em relação ao modelo de atenção (ESF ou tradicional). Equipes responsáveis por mais de 4000 moradores apresentaram menor prevalência de adequação $(\mathrm{RP}=0,88$; IC95\%=0,83-0,94) do que as equipes com até 4000 moradores. A prevalência de adequação do processo de trabalho foi maior entre as unidades que contavam com equipe mínima completa em relação àquelas unidades que não possuíam equipe completa ( $\mathrm{RP}=1,08$; IC $95 \%=1,01$ 1,16) (Tabela 2).

Comparadas à Região Norte (24\%), as Regiões Sudeste (39\%) e Sul (31\%) apresentaram maior prevalência de equipes com processo de trabalho adequado, enquanto que a Região Centro-Oeste apresentou o menor percentual de unidades classificadas como adequadas (18\%). Os municípios com mais de 100.000 habitantes mostraram proporções elevadas de adequação, e quanto maior o IDH, melhores também as prevalências de adequação. Em contrapartida, a prevalência de adequação do processo de trabalho foi menor nas áreas com maior coberturas da ESF (Tabela 2).

Observou-se que a proporção de UBS com equipes que possuíam processo de trabalho adequado foi $35 \%$ maior entre as UBS com estrutura adequada $(\mathrm{RP}=1,35 ; \mathrm{IC} 95 \%=1,28-1,42)$.

\section{Tabela 2}

Prevalência e razões de prevalência para adequação da estrutura das unidades básicas e do processo de trabalho das equipes referentes ao exame preventivo do câncer de colo de útero. Avaliação externa do PMAQ-AB, Brasil, 2012.

\begin{tabular}{|c|c|c|c|c|}
\hline Variável & $\begin{array}{c}\text { Adequação } \\
\text { da estrutura (\%) }\end{array}$ & RP (IC95\%) & $\begin{array}{c}\text { Adequação } \\
\text { do processo (\%) }\end{array}$ & RP (IC95\%) \\
\hline \multicolumn{5}{|l|}{ Modelo de atenção* } \\
\hline Tradicional & 36,3 & 1,00 & 31,0 & 1,00 \\
\hline Saúde da Família & 53,0 & $1,35(1,33-1,38)$ & 30,3 & $0,98(0,86-1,11)$ \\
\hline \multicolumn{5}{|c|}{ US participante do PMAQ\# } \\
\hline Não & 42,7 & 1,00 & -- & -- \\
\hline Sim & 61,2 & $1,43(1,41-1,46)$ & & \\
\hline \multicolumn{5}{|c|}{ População adscrita à equipe\#\# (hab.) } \\
\hline Até 4000 & -- & -- & 31,2 & 1,00 \\
\hline Mais de 4000 & & & 27,6 & $0,88(0,83-0,94)$ \\
\hline \multicolumn{5}{|c|}{ Equipe mínima de SF completa\#\# } \\
\hline Não & -- & -- & 28,2 & 1,00 \\
\hline Sim & & & 30,6 & $1,08(1,01-1,16)$ \\
\hline \multicolumn{5}{|l|}{ Região } \\
\hline Norte & 37,3 & 1,00 & 24,3 & 1,00 \\
\hline Nordeste & 39,6 & $1,06(1,01-1,12)$ & 22,8 & $0,94(0,83-1,06)$ \\
\hline Sudeste & 55,9 & $1,50(1,43-1,57)$ & 39,3 & $1,62(1,45-1,81)$ \\
\hline Sul & 60,8 & $1,63(1,55-1,71)$ & 30,7 & $1,26(1,12-1,43)$ \\
\hline Centro-Oeste & 59,9 & $1,61(1,52-1,70)$ & 17,8 & $0,73(0,62-0,87)$ \\
\hline
\end{tabular}


Prevalência e razões de prevalência para adequação da estrutura das unidades básicas e do processo de trabalho das equipes referentes ao exame preventivo do câncer de colo de útero. Avaliação externa do PMAQ-AB, Brasil, 2012.

\begin{tabular}{|c|c|c|c|c|}
\hline Variável & $\begin{array}{c}\text { Adequação } \\
\text { da estrutura (\%) }\end{array}$ & RP (IC95\%) & $\begin{array}{c}\text { Adequação } \\
\text { do processo (\%) }\end{array}$ & RP (IC95\%) \\
\hline \multicolumn{5}{|c|}{ Porte populacional (hab.) } \\
\hline Até 10.000 & 48,4 & 1,00 & 27,5 & 1,00 \\
\hline 10.001 a 30.000 & 46,0 & $0,95(0,92-0,98)$ & 25,6 & $0,93(0,86-1,00)$ \\
\hline 30.001 a 100.000 & 47,9 & $0,99(0,96-1,02)$ & 26,7 & $0,97(0,90-1,06)$ \\
\hline 100.001 a 300.000 & 55,3 & $1,14(1,10-1,18)$ & 30,8 & $1,12(1,03-1,23)$ \\
\hline Mais de 300.000 & 55,0 & $1,14(1,10-1,18)$ & 40,0 & $1,46(1,36-1,57)$ \\
\hline \multicolumn{5}{|l|}{ IDH-M\& } \\
\hline 0,467 a 0,642 & 38,0 & 1,00 & 21,5 & 1,00 \\
\hline 0,643 a 0,730 & 43,3 & $1,14(1,10-1,18)$ & 25,7 & $1,20(1,10-1,30)$ \\
\hline 0,731 a 0,787 & 53,2 & $1,40(1,36-1,44)$ & 27,1 & $1,26(1,16-1,38)$ \\
\hline 0,788 a 0,919 & 62,8 & $1,65(1,60-1,70)$ & 40,1 & $1,87(1,73-2,01)$ \\
\hline \multicolumn{5}{|l|}{ Cobertura de ESF (\%) } \\
\hline até 50 & 51,4 & 1,00 & 38,4 & 1,00 \\
\hline 50,1 a 75 & 50,3 & $0,98(0,95-1,01)$ & 27,4 & $0,71(0,67-0,76)$ \\
\hline 75,1 a 99,9 & 48,7 & $0,95(0,92-0,97)$ & 26,2 & $0,68(0,64-0,73)$ \\
\hline 100 & 46,4 & $0,90(0,89-0,93)$ & 27,1 & $0,71(0,67-0,75)$ \\
\hline Total & 49,3 & & $30,3 \%$ & \\
\hline
\end{tabular}

\# Informação disponível apenas para as UBS - Módulo I (todas as equipes avaliadas participaram do PMAQ);

\#\# 1 Médico + 1 enfermeiro + 1 auxiliar ou técnico de enfermagem + 4 agentes comunitários de saúde - Informação disponível apenas para as equipes - Módulo $\mathrm{II}_{i}$

\& Índice de Desenvolvimento Humano Municipal;

* Unidade de Saúde com Estratégia de Saúde da Família.

\section{Discussão}

Os resultados mostraram que apenas metade das unidades de saúde da rede básica no Brasil apresentou estrutura adequada para o rastreamento do câncer de colo de útero por meio do exame citopatológico e somente $30 \%$ das equipes foram classificadas com processo de trabalho adequado para detecção do câncer de colo de útero. Recente estudo identificou diversas barreiras à integralidade do cuidado ao câncer de colo principalmente o acesso e a qualidade da atenção. 15

Vários outros estudos que investigaram a qualidade dos serviços prestados na rede básica de saúde e, via de regra, identificaram uma ampla gama de deficiências, como relatado por Assunção et al.,16 ao avaliar a rede na atenção aos diabéticos, de Costa et al. 17 em relação ao pré-natal, de Saparolli et al. ${ }^{18}$ ao avaliar a atenção à criança dispensada pela enfermagem e de Costa et al.19 para a saúde da criança na Estratégia Saúde da Família. Estudo que comparou o desempenho da ESF em relação ao modelo de assistência tradicional nas Regiões Sul e Nordeste, não encontrou diferença na estrutura física entre estas unidades, que foram identificadas como precárias e improvisadas. 20

A ausência de alguns dos itens não impede a realização da coleta de material e do exame histopatológico, como por exemplo, o foco de luz ou a ficha de requisição do sistema de informações específico (Siscolo). Entretanto, a falta de fixador de lâmina em mais de um terço das Unidades e de espéculo e espátula em $20 \%$ delas inviabiliza a realização do procedimento. Além disso, apenas unidades de saúde que disponham de todos os equipamentos e insumos avaliados podem cumprir integralmente os procedimentos desta ação programática, uma vez que a disponibilidade do conjunto de itens elencados pode ser considerada como situação ideal para a coleta do exame no âmbito de uma unidade de saúde.

A prevalência de adequação da estrutura foi maior entre as UBS cujo modelo assistencial era a ESF e entre aquelas que haviam aderido ao PMAQ. Os dados sugerem que a adoção da ESF como 
modelo de atenção básica parece contribuir para a melhoria da estrutura dos serviços de saúde e, consequentemente, para a prestação de serviços voltados à prevenção do câncer de colo uterino. Por outro lado, esse resultado pode estar refletindo o reforço das unidades na aquisição dos equipamentos e insumos necessários ao desenvolvimento das ações de prevenção ao câncer de colo pelos gestores e equipes de saúde antes da realização da visita pela equipe da pesquisa, uma vez que os mesmos foram previamente avisados da realização deste estudo.

De qualquer forma, deve-se considerar que esse possível viés pode ter trazido benefícios à assistência à saúde da população, uma vez que propiciou melhorias na qualidade da prestação deste serviço. Este seria um aspecto positivo do processo de avaliação como um todo, pois induziu melhorias não apenas na estrutura dos serviços de saúde, mas também em relação às equipes. Assim, conclui-se que a realização deste estudo de avaliação do PMAQ-AB tenha trazido benefícios para as US e para as equipes, uma vez que o processo promoveu melhorias na estrutura e no funcionamento das US e na qualificação do processo de trabalho. 11

Entre os itens avaliados do processo de trabalho das equipes, destaca-se o fato de apenas $45 \%$ delas realizarem o registro das coletas de material e de exames de Papanicolau. A escassez de sistemas de registro de dados relativos à assistência na atenção básica é um problema reconhecido, o qual vem merecendo cada vez mais esforços por parte dos gestores de saúde. Se $100 \%$ das equipes realizassem o registro dos exames, a adequação do processo de trabalho teria se elevado de $30 \%$ para $53 \%$, destacando-se a importância deste indicador para a melhoria da atenção ao evento estudado. Tornam-se, portanto, necessários mais investimentos em tecnologia de informática e comunicação e para a capacitação dos profissionais de saúde. ${ }^{21}$

Os dados do estudo evidenciam as acentuadas desigualdades regionais relacionadas à situação de saúde em nosso país 22 e enfatizam as iniquidades no âmbito da atenção básica de saúde. As Regiões Norte e Nordeste apresentaram os piores resultados tanto com relação à adequação da estrutura quanto ao processo de trabalho das equipes. Chamou a atenção o baixo percentual de adequação do processo de trabalho entre as equipes de saúde da região CentroOeste, considerando o fato de se ter observado maiores percentuais de adequação da estrutura das unidades de saúde nas quais atuavam.

Em relação ao porte dos municípios e seu IDH, observou-se que os municípios com mais de 100.000 habitantes e aqueles com maior IDH apresentavam maior frequência de adequação quanto à estrutura e processo de trabalho. Esse resultado reflete as melhores condições econômicas dos grandes centros urbanos, com maior capacidade de investimento e de capacitação das equipes de saúde. 23

Constatou-se que quanto maior a cobertura populacional da ESF no município, menor o percentual de adequação das unidades de saúde, tanto em relação à estrutura quanto às rotinas para a realização do exame de Papanicolau. Este resultado pode ser atribuído ao fato de os pequenos municípios concentrarem as maiores coberturas da ESF, sendo em geral os que apresentam menor IDH. ${ }^{24} \mathrm{Um}$ dos componentes do IDH é justamente o produto interno bruto per capita, podendo-se inferir que municípios menores têm menor capacidade de investimento em saúde do que os maiores. Assim, tendem a dispor de serviços de saúde com estrutura mais deficitária.25,26

Entretanto, ao se considerar a situação de cada unidade de saúde, independentemente da cobertura de saúde da família do município, constatou-se que a situação é inversa nas unidades de saúde da ESF, fato que pode ser explicado pela peculiaridade do processo de expansão da ESF no Brasil, que foi inicialmente implantado nos municípios de menor porte. 20,26 Conclui-se, portanto, que caso a cobertura do ESF atingisse $100 \%$ da população dos municípios, independentemente do seu porte e nível de IDH, a qualidade das ações assistenciais e de prevenção se elevariam a níveis mais satisfatórios, a exemplo dos resultados deste estudo.

O Ministério da Saúde, no documento que normatiza o PMAQ, reconhece a precariedade da rede física das UBS e afirma a necessidade de qualificação dos processos de trabalho das equipes de atenção básica, o que inclui integração dos membros das equipes, orientação do trabalho em função de prioridades, metas e resultados. Problemas como a instabilidade das equipes e elevada rotatividade dos profissionais ainda comprometem o vínculo, a continuidade do cuidado e a integração da equipe. Além disso, a sobrecarga das equipes com número excessivo de pessoas sob sua responsabilidade pode ter impacto na cobertura e na qualidade de suas ações. 11,27

Considerando que o exame de prevenção de câncer cervicouterino deve ter cobertura universal para mulheres entre 25 e 64 anos de idade, espera-se que a rede básica de saúde deva estar preparada para a realização do exame e assuma importante papel na disseminação de informação e esclarecimentos da população sobre os benefícios do rastreamento na prevenção do câncer de colo uterino. Além disso, a rede básica assume papel relevante na identificação 
e encaminhamento da população com maior risco de câncer de colo e a elegível para a realização do exame cervico-uterino na unidade de saúde, além da realização de busca ativa e identificação de mulheres faltosas, entre outras ações. 8,28 Dessa forma, acredita-se que o processo de avaliação das ações de saúde desenvolvidas pelas unidades participantes do PMAQ possa ser oportuno na indução de mudanças nas condições, no funcionamento das unidades de saúde e na qualificação de suas equipes de saúde. 11

Neste estudo, foi possível examinar a estrutura e o processo de trabalho no âmbito da ação programática de prevenção e controle do câncer de colo de útero, tendo-se constatado maior adequação do processo de trabalho entre as US com melhor estrutura. Donabedian ${ }^{29}$ recomendava este tipo de estudo de avaliação como meio de se estimar a qualidade global dos serviços de saúde. $30 \mathrm{O}$ autor destaca as dificuldades em se promover mudanças no comportamento das equipe e sugere que tais mudanças podem estar relacionadas à estrutura do serviço e ao processo de trabalho. Donabedian igualmente propõe que o pior desempenho do serviço de saúde nem sempre está relacionado à competência individual dos componentes da equipe, mas também ao contexto. Em que pese o recorte aqui abordado, acredita-se que este tipo de análise possa reforçar as evidências e estimular a melhoria da estrutura para todos os programas desenvolvidos na rede básica.

Os desfechos analisados deste estudo foram construídos com base nas informações disponíveis

\section{Referências}

1. International Agency for Research on Cancer. GLOBOCAN 2012: Estimated cancer incidence, mortality and prevalence worldwide in 2012. 2012 [cited 2014 April 29]; Available from: http://globocan.iarc.fr/Pages/fact_sheets_population.aspx

2. Instituto Nacional do Câncer. Atlas da Mortalidade. 2012; Available from: http://mortalidade.inca.gov.br/Mortalidade Web.

3. Brasil. Ministério da Saúde. Secretaria Nacional de Assistência à Saúde. Falando sobre câncer do colo do útero. Rio de Janeiro: Instituto Nacional de Câncer. Coordenação de Prevenção e Vigilância (Conprev)2002.

4. Quadros CAT, Victora CG, Dias da Costa JS. Coverage and focus of a cervical cancer prevention program in southern Brazil. Rev Panam Salud Publica 2004; 16: 223-32.

5. WHO (World Health Organization). Comprehensive cervical cancer control: a guide to essential practice. Geneva; 2007.

6. DATASUS. Sistema de Gerenciamento da Tabela de Procedimentos, Medicamentos e OPM do SUS. 2014 [20 de dezembro de 2014]; Available from: nos módulos utilizados para avaliação externa do PMAQ. A avaliação de informações igualmente importantes, como a qualidade dos registros e dos insumos disponíveis, além das condições de ambiência física específicas à realização dos exames não foram contemplados neste estudo. Entretanto, deve-se destacar a abrangência nacional do estudo, o qual incluiu UBS de áreas urbanas e rurais. Os dados da estrutura foram censitários, constituindo um trabalho de campo sem precedentes na história do país. A eventual dificuldade de padronização na coleta pelo grande número de entrevistadores foi minimizada pelos intensos treinamentos para a observação e verificação dos documentos comprobatórios. Além disso, o instrumento eletrônico continha regras de validação embutidas, saltos sistemáticos para os casos de perguntas que não se aplicassem, além do controle de tempo de aplicação e a proporção de variáveis com respostas ignoradas.

Conclui-se que, de um modo geral, para que se alcance maior efetividade nas ações de prevenção ao câncer do colo do útero no Brasil, faz-se necessária a melhoria dos níveis de adequação desta ação programática na rede básica de saúde, havendo a necessidade de se promover melhorias substanciais, principalmente o aperfeiçoamento e implantação de sistemas de registro das atividades desenvolvidas na UBS, a qualificação das equipes e o aumento do aporte de insumos e materiais necessários ao pleno desenvolvimento das ações. http://sigtap.datasus.gov.br/tabela-unificada/app/sec/inicio. jsp.

7. Instituto Nacional do Câncer. Agenda estratégica para o controle do câncer do colo do útero. 2014; Available from: http://www2.inca.gov.br/wps/wcm/connect/acoes_program as/site/home/agenda_estrategica/apresentacao_agenda_estr ategica.

8. Brasil. Ministério da Saúde. Controle dos cânceres do colo do útero e da mama. (Cadernos de Atenção Básica, n. 13). In: Departamento de Atenção Básica, editor. Brasília: Editora do Ministério da Saúde; 2013. p. 124.

9. IBGE (Instituto Brasileiro de Geografia e Estatística). Pesquisa Nacional por Amostra de Domicílios: acesso e utilização de serviços, condições de saúde e fatores de risco e proteção à saúde 20082010 Contract $\mathrm{N}^{\circ}$ : ISBN 978-85240-4112-9.

10. Cesar JA, Horta BL, Gomes G, Houlthausen RS, Willrich RM, Kaercher A, Iastrenski FM. Fatores associados à não realização de exame citopatológico de colo uterino no extremo Sul do Brasil. Cad Saúde Pública. 2003; 19: 136572. 
11. Brasil. Ministério da Saúde. Saúde mais perto de você Programa Nacional da Melhoria do Acesso e da Qualidade da Atenção Básica. Manual Instrutivo: Secretaria de Atenção à Saúde. Departamento de Atenção Básica; 2012.

12. IBGE (Instituto Brasileiro de Geografia e Estatística) Censo demográfico 2010. 2012; Available from: http://www.ibge.gov.br/home/estatistica/populacao/censo20 $10 /$ default.shtm

13. Programa das Nações Unidas para o Desenvolvimento. Índice de Desenvolvimento Humano Municipal. 2010 [7 mai 2014]; Available from: http://www.pnud.org.br/IDH/ Default.aspx?indiceAccordion $=1 \& l i=1 i$ AtlasMunicipios

14. Brasil Ministério da Saúde. Política Nacional de Atenção Básica. In: Departamento de Atenção Básica, editor. Brasília; 2012

15. Brito-Silva K, Bezerra AFB, Chaves LDP, Tanaka OY. Integralidade no cuidado ao câncer do colo do útero: avaliação do acesso. Rev Saúde Pública. 2014; 48: 240-8

16. Assunção MC, Santos IS, Gigante DP. Atenção primária em diabetes no Sul do Brasil: estrutura, processo e resultado. Rev Saúde Pública. 2001; 35: 88-95.

17. Costa GD, Cotta RMM, Reis JR, Siqueira-Batista R, Gomes AiPc, Franceschini SCC. Avaliação do cuidado à saúde da gestante no contexto do Programa Saúde da Família. Ciênc Saúde Coletiva. 2009; 14: 1347-57.

18. Saparolli ECL, Adami NP. Avaliação da estrutura destinada à consulta da enfermagem à criança na atenção básica. Rev Esc Enferm USP. 2010; 44: 92-8.

19. Costa GD, Cotta RMM, Reis JR, Ferreira MLSM, Reis RS, Franceschini SCC. Avaliação da atenção à saúde da criança no contexto da Saúde da Família no município de Teixeiras, Minas Gerais (MG, Brasil). Ciênc Saúde Coletiva. 2011; 16: 3229-40.

20. Facchini LA, Piccini RX, Tomasi E, Thumé E, Silveira DS Siqueira FV, Rodrigues Ma. Desempenho do PSF no Sul e no Nordeste do Brasil: avaliação institucional e epidemiológica da Atenção Básica à Saúde. Ciênc Saúde Coletiva 2006; 11: 669-81.

21. Instituto Nacional de Câncer. Sistema de informação do controle do câncer de mama (Sismama) e do câncer do colo

Recebido em 22 de dezembro 2014

Versão final apresentada em 12 de março de 2015

Aprovado em 27 de março de 2015 do útero (Siscolo): manual gerencial In: Coordenação Geral de Ações Estratégicas. Divisão de Apoio à Rede de Atenção Oncológica, editor. Rio de Janeiro; 2011.

22. Duarte EC, Schneider MC, Paes-Sousa R, Ramalho WM, Sardinha LV, Silva Júnior JB, Castillo-Salgado C. Epidemiologia das desigualdades em saúde no Brasil: um estudo exploratório. Brasília: Organização Pan-Americana da Saúde; 2002.

23. Viana ALA, Rocha JSY, Elias PE, Ibañez N, Novaes MH. Modelos de atenção básica nos grandes municípios paulistas: efetividade, eficácia, sustentabilidade e governabilidade. Ciênc Saúde Coletiva. 2006; 11: 577-606.

24. Programa das Nações Unidas para o Desenvolvimento. Evolução do desenvolvimento humano nos municípios brasileiros. 2014 [cited 2014]; Available from: www.pnud.org.br.

25. Henrique F, Calvo MCM. Grau de implantação do Programa Saúde da Família e indicadores sociais. Ciênc Saúde Coletiva. 2009; 14: 1359-65.

26. Brasil. Departamento de Atenção Básica. Pesquisa nacional registra a evolução do Saúde da Família e sua relação com indicadores de saúde. 2005; Available from: http://189.28.128.100/dab/docs/publicacoes/informes/psfin fo32.pdf.

27. Loch-Neckel G, Seemann G, Eidt HB, Rabuske MM, Crepaldi MA. Desafios para a ação interdisciplinar na atenção básica: implicações relativas à composição das equipes de saúde da família. Ciênc Saúde Coletiva. 2009; 14: 1463-72.

28. Spadea T, Bellini S, Kunst A, Stirbu I, Costa G. The impact of interventions to improve attendance in female cancer screening among lower socioeconomic groups: a review. Prev Med. 2010; 50: 159-64.

29. Donabedian A. The quality of care. How can it be assessed? JAMA. 1988; 260: 1743-8.

30. Silveira DS, Santos IS, Dias da Costa JS. Atenção pré-natal na rede básica: uma avaliação da estrutura e do processo. Cad Saúde Pública. 2001; 17: 131-9. 